\title{
Osimertinib-induced interstitial lung disease in a patient with non-small cell lung cancer pretreated with nivolumab: A case report
}

\author{
OSAMU TAKAKUWA, TETSUYA OGURI, TAKEHIRO UEMURA, KAZUKI SONE, \\ SATOSHI FUKUDA, MINAMI OKAYAMA, YOSHIHIRO KANEMITSU, HIROTSUGU OHKUBO, \\ MASAYA TAKEMURA, YUTAKA ITO, KEN MAENO and AKIO NIIMI \\ Department of Respiratory Medicine, Allergy and Clinical Immunology, \\ Nagoya City University Graduate School of Medical Sciences, Nagoya, Aichi 467-8601, Japan
}

Received January 19, 2017; Accepted July 4, 2017

DOI: $10.3892 / \mathrm{mco} .2017 .1349$

\begin{abstract}
Osimertinib (AZD9291) is a third-generation epidermal growth factor receptor (EGFR) tyrosine kinase inhibitor approved for EGFR-T790M-positive non-small cell lung cancer. A high incidence of interstitial lung disease (ILD) during combination treatment with osimertinib and anti-programmed cell death-ligand 1 (PD-L1) inhibitor has been reported. The current study presents a case of ILD development during osimertinib treatment following nivolumab (an anti-PD-1 antibody) treatment. The 59-year-old female was diagnosed with stage IV lung adenocarcinoma harboring a deletion in exon 19 of the EGFR gene. Following nivolumab as a sixth-line treatment, an EGFR-T790M-encoding mutation in EGFR exon 20 was identified by re-biopsy. Osimertinib was therefore initiated as a seventh-line treatment. A partial response was subsequently noted; however, 63 days after initiation of the treatment the patient presented with dyspnea with decreased oxygenation in the absence of fever and sputum. A computed tomography scan revealed the emergence of ground-glass opacities with bronchiectasis in both lungs, and a diagnosis of ILD due to osimertinib was made. Following steroid pulse therapy with discontinuation of osimertinib, the patient's chest findings and respiratory condition improved. Therefore, it is considered that anti-PD-1 therapies may be associated with a risk of ILD during subsequent osimertinib treatment.
\end{abstract}

Correspondence to: Dr Tetsuya Oguri, Department of Respiratory Medicine, Allergy and Clinical Immunology, Nagoya City University Graduate School of Medical Sciences, 1 Kawasumi, Mizuho-cho, Mizuho-ku, Nagoya, Aichi 467-8601, Japan

E-mail: t-oguri@med.nagoya-cu.ac.jp

Key words: osimertinib, interstitial lung disease, nivolumab, non-small-cell lung cancer, epidermal growth factor receptor tyrosine kinase inhibitor

\section{Introduction}

The discovery of epidermal growth factor receptor (EGFR)-activating mutations in non-small cell lung cancer (NSCLC) and EGFR tyrosine kinase inhibitors (TKIs) have changed the strategy of NSCLC therapy. Recently, EGFR-TKIs, including gefitinib, erlotinib and afatinib, have become the standard therapy for patients with advanced EGFR-mutated NSCLC. However, the majority of the patients progress and second-line therapy is required.

The EGFR T790M point mutation (T790M) is the most common mechanism underlying drug resistance to EGFR-TKIs in EGFR mutation-positive NSCLC patients. Osimertinib (AZD9291) is a third-generation EGFR-TKI approved for the treatment of EGFR-T790M-positive NSCLC (1). Furthermore, immune checkpoint modulation with programmed death-1 (PD-1) or programmed death-ligand 1 (PD-L1) inhibition has also shown promise in changing the strategy of NSCLC therapy. Nivolumab, an anti-PD-1 inhibitor, is a novel drug used in the second-line treatment of NSCLC (2). In both EGFR-TKI and immune checkpoint inhibitors therapy, interstitial lung disease (ILD) is recognized as one of most severe adverse events. These two therapies may be sequentially applied in the same patient, but the effects of the interaction between these two drugs on the risk of ILD remains unclear. A case of ILD that occurred during osimertinib treatment in a patient with a history of previous nivolumab treatment is reported herein.

\section{Case report}

A 59-year-old female was diagnosed with stage IV (cT3N2M1a) lung adenocarcinoma harboring a deletion in exon 19 of the EGFR gene. The patient was treated with platinum-pemetrexed with bevacizumab, followed by maintenance therapy for 6 months. Subsequently, the patient received third-line treatment with afatinib for 6 months, fourth-line S-1 for 5 months, fifth-line docetaxel for 2 months and sixth-line nivolumab for 1 month (administered twice). Eventually, 2.5 years after the initial diagnosis, the patient's disease progressed and a computed tomography (CT)-guided 

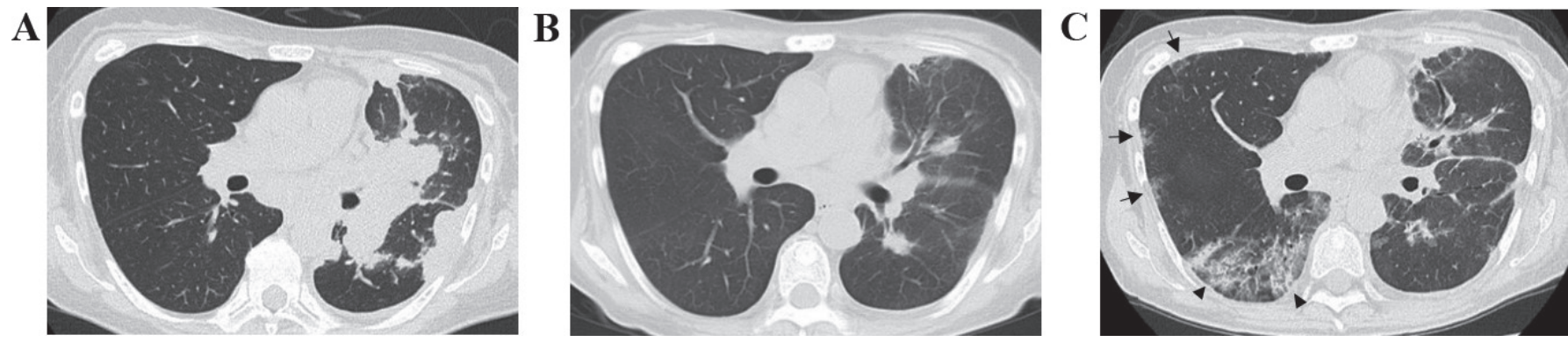

Figure 1. CT findings in the present case. (A) Chest CT scan prior to osimertinib treatment. Small nodules in the intermediate and right lower lung lobes are observed. (B) A chest CT scan 50 days after osimertinib treatment initiation. Shrinking of the nodules in the right field is observed. (C) Chest CT scan 63 days after osimertinib treatment initiation. Patchy ground-glass opacities (arrows) and consolidation with bronchiectasis (arrowheads) may be seen in the right intermediate and lower lung lobes. CT, computed tomography.

percutaneous needle biopsy for chest wall invasion revealed an EGFR-T790M-encoding mutation in EGFR exon 20. A total of 37 days after the last initiation of nivolumab, oral osimertinib therapy was initiated at a dose of $80 \mathrm{mg}$ per day. Two months later, a chest $\mathrm{CT}$ revealed a partial response (Fig. 1A and B) and the patient's performance status (PS) improved from 3 to 1 . However, on the 63rd day after osimertinib initiation, the patient presented to the hospital with shortness of breath. The patient had a light cough, without sputum or fever. A chest X-ray revealed ground-glass shadows in the lungs bilaterally and a chest $\mathrm{CT}$ showed the emergence of ground-glass opacities and consolidation with bronchiectasis in the right middle and lower lung lobes (Fig. 1C). The patient's peripheral capillary oxygen saturation was $96 \%$, which was lower compared with that at the previous visit, and the laboratory findings revealed increased levels of lactate dehydrogenase (427 IU/1; normal range, 119-229 IU/1) and C-reactive protein $(1.43 \mathrm{mg} / \mathrm{dl}$; normal range, $<0.30 \mathrm{mg} / \mathrm{dl})$. The patient was hospitalized with a suspected diagnosis of ILD induced by osimertinib, and was treated with steroid pulse therapy, antibiotics (levofloxacin) and discontinuation of osimertinib. Following treatment with steroid pulse therapy, the patient's chest findings and respiratory condition improved and the steroid therapy was switched to prednisolone and the dose was reduced. The serum laboratory tests, including tests for antibodies against atypical pneumonia and immune rheumatic disease, $\beta$-D glucan and cytomegalovirus antigen, were all negative. After 10 days of hospitalization, the patient was discharged from the hospital with continuous steroid therapy of $10 \mathrm{mg}$ prednisolone. Subsequently, the ILD remained in remission but the cancer rapidly progressed. Re-treatment with osimertinib and other chemotherapy was not administered; instead, opioid therapy was initiated. Twenty days following hospitalization for ILD, the patient succumbed to cancer progression.

Informed consent was obtained from the patient regarding the publication of the case details and associated images.

\section{Discussion}

For patients with advanced EGFR-mutated NSCLC, treatment with first- or second-generation EGFR-TKIs is associated with response rates of 56-74\% (3-6), but the majority of the patients develop disease progression within 1-2 years following treatment initiation (3-6). In $\sim 60 \%$ of the patients, the mechanism underlying the acquired resistance is the development of an additional EGFR mutation, encoding EGFR T790M (7).

Osimertinib treatment has demonstrated a response rate of $>60 \%$, with durable progression-free survival for NSCLC patients with the EGFR-T790M-resistance mutation (1). The incidence of interstitial pneumonia during osimertinib treatment was reported to be $3 \%$, and grade 5 was observed in $1 \%$ of the cases (1). As osimertinib is used in pretreated patients, greater attention must be paid to the incidence of interstitial pneumonia induced by osimertinib compared with other EGFR-TKIs.

It is known that risk factors of ILD in EGFR-TKI treatment include a history of smoking, concomitant interstitial pneumonia and a poor PS (8). In the present study, a poor PS may have been the reason for the incidence of ILD. On the other hand, Ahn et al (9) reported that ILD was observed in $38 \%$ of cases receiving combination treatment with osimertinib and durvalumab, a PD-L1 inhibitor, which suggests that treatment with such a drug combination may dramatically increase the risk of ILD. Notably, a similar increased risk of ILD has not been reported for combination treatment with durvalumab and gefitinib (10). These studies suggest that previous treatment with nivolumab may affect the onset of ILD during osimertinib treatment. Durability of the response following discontinuation of nivolumab has been observed (11). As previous treatment with nivolumab may be a critical risk factor for ILD during osimertinib treatment, further verification of this possibility is required.

In sub-group analysis in a previous phase III study (2), it was reported that nivolumab was relatively inferior to docetaxel in patients harboring an EGFR mutation. Furthermore, a meta-analysis reported that immune-checkpoint inhibitors do not improve overall survival over docetaxel in EGFR-mutated advanced NSCLC (12). We consider that nivolumab treatment prior to osimertinib treatment should be considered with caution when devising a treatment strategy for patients with EGFR-mutated advanced NSCLC. The presence of PD-L1 expression has been reported to be a predictive biomarker of the efficacy of anti-PD-1/PD-L1 antibodies, including nivolumab (13). Assessment of tumor PD-L1 expression may aid with decision-making regarding the adoption of nivolumab treatment in patients with EGFR-mutated advanced NSCLC.

In conclusion, the present study reports a case of ILD during osimertinib treatment following treatment with nivolumab. Careful assessment is required prior to commencing osimertinib 
treatment in patients previously treated with nivolumab. Further studies investigating the risk of ILD during EGFR-TKI treatment following nivolumab pre-treatment are required to reach more definitive conclusions.

\section{Acknowledgements}

Tetsuya Oguri and Akio Niimi have received personal fees from ONO PHARMACEUTICAL Co., Ltd. (Osaka, Japan), and AstraZeneca (Osaka, Japan). Ken Maeno has received grants from AstraZeneca. Masaya Takemura has received grants and personal fees from AstraZeneca and personal fees from ONO PHARMACEUTICAL Co., Ltd.

\section{References}

1. Jänne PA, Yang JC,Kim DW,PlanchardD, Ohe Y,Ramalingam SS Ahn MJ, Kim SW, Su WC, Horn L, et al: AZD9291 in EGFR inhibitor-resistant non-small cell lung cancer. N Engl J Med 372: $1689-1699,2015$

2. Brahmer J, Reckamp KL, Baas P, Crinò L, Eberhardt WE, Poddubskaya E, Antonia S, Pluzanski A, Vokes EE, Holgado E, et al: Nivolumab versus docetaxel in advanced squamous-cell non-small cell lung cancer. N Engl J Med 373: $123-135,2015$

3. Mok TS, Wu YL, Thongprasert S, Yang CH, Chu DT, Saijo N, Sunpaweravong P, Han B, Margono B, Ichinose Y, et al: Gefitinib or carboplatin-paclitaxel in pulmonary adenocarcinoma. N Engl J Med 361: 947-957, 2009.

4. Maemondo M, Inoue A, Kobayashi K, Sugawara S, Oizumi S, Isobe H, Gemma A, Harada M, Yoshizawa H, Kinoshita I, et al: Gefitinib or chemotherapy for non-small-cell lung cancer with mutated EGFR. N Engl J Med 362: 2380-2388, 2010.

5. Rosell R, Carcereny E, Gervais R, Vergnenegre A, Massuti B, Felip E, Palmero R, Garcia-Gomez R, Pallares C, Sanchez JM, et al: Erlotinib versus standard chemotherapy as first-line treatment for European patients with advanced EGFR mutation-positive non-small-cell lung cancer (EURTAC): A multicentre, open-label, randomised phase 3 trial. Lancet Oncol 13: 239-246, 2012.

6. Sequist LV, Yang JC, Yamamoto N, O'Byrne K, Hirsh V, Mok T, Geater SL, Orlov S, Tsai CM, Boyer M, et al: Phase III study of afatinib or cisplatin plus pemetrexed in patients with metastatic lung adenocarcinoma with EGFR mutations. J Clin Oncol 31 $3327-3334,2013$
7. Yu HA, Arcila ME, Rekhtman N, Sima CS, Zakowski MF, Pao W, Kris MG, Miller VA, Ladanyi M and Riely GJ: Analysis of tumor specimens at the time of acquired resistance to EGFR-TKI therapy in 155 patients with EGFR-mutant lung cancers. Clin Cancer Res 19: 2240-2247, 2013.

8. Hotta K, Kiura K, Takigawa N, Yoshioka H, Harita S, Kuyama S, Yonei T, Fujiwara K, Maeda T, Aoe K, et al: Comparison of the incidence and pattern of interstitial lung disease during erlotinib and gefitinib treatment in Japanese Patients with non-small cell lung cancer: The Okayama Lung Cancer Study Group experience. J Thorac Oncol 5: 179-184, 2010

9. Ahn MJ, Yang J, Yu H, Saka H, Ramalingam S, Goto K, Kim SW, Yang L, Walding A and Oxnard GR: 136O: Osimertinib combined with durvalumab in EGFR-mutant non-small cell lung cancer: Results from the TATTON phase Ib trial. J Thorac Oncol 11: S115, 2016.

10. Gibbons DL, Chow LQ, Kim DW, Kim SW, Yeh T, Song X, Jiang H, Taylor R, Karakunnel J and Creelan B: 570 Efficacy, safety and tolerability of MEDI4736 (durvalumab [D]), a human IgG1 anti-programmed cell death-ligand-1 (PD-L1) antibody, combined with gefitinib (G): A phase I expansion in TKI-naïve patients (pts) with EGFR mutant NSCLC. J Thorac Oncol 11: S79, 2016.

11. Gettinger SN, Horn L, Gandhi L, Spigel DR, Antonia SJ, Rizvi NA Powderly JD, Heist RS, Carvajal RD, Jackman DM, et al: Overall survival and long-term safety of nivolumab (anti-programmed death 1 Antibody, BMS-936558, ONO-4538) in patients with previously treated advanced non-small-cell lung cancer. J Clin Oncol 33: 2004-2012, 2015.

12. Lee CK, Man J, Lord S, Links M, Gebski V, Mok T and Yang JC: Checkpoint inhibitors in metastatic EGFR-mutated non-small cell lung cancer-A meta-analysis. J Thorac Oncol 12: 403-407, 2017.

13. Zhang T, Xie J, Arai S, Wang L, Shi X, Shi N, Ma F, Chen S, Huang L, Yang L, et al: The efficacy and safety of anti-PD-1/PD-L1 antibodies for treatment of advanced or refractory cancers: A meta-analysis. Oncotarget 7: 73068-73079, 2016. 